\title{
CONDUCTIVITY OF GRAINS IN NONLINEAR OXIDE CERAMICS AND PROTECTIVE PROPERTIES OF VARISTORS
}

\author{
A.I. Ivon*, R.I. Lavrov \\ Oles Honchar Dnipro National University, Dnipro, Ukraine \\ e-maila_ivon@mail.ru
}

The current-voltage characteristics of various compositions of nonlinear varistor ceramics based on $\mathrm{ZnO}$ (ZOC) and $\mathrm{SnO}_{2}$ (TOC) are studied in a wide range of electric current. On the basis of obtained data were determined the parameters that allow finding the coefficients $k_{p r s}$ and $k_{p r l}$, which define the protection levels against the switching surge and the lightning surge for surge suppressors on the basis of $\mathrm{ZOC}$ and TOC varistors. The lower the value of these coefficients are, the significantly higher the efficiency of surge protection is. A relationship between $k_{p r s}, k_{p r l}$, and the effective linear resistivity $\rho_{\text {lin }}$ limiting the current of intergranular barriers in varistor ceramics are established. It is shown that the values of $k_{p r s}$ and $k_{p r l}$ increase with increasing $\rho_{l i n}$, which value is determined by the bulk resistance of the ceramic grains. The studied TOC compositions have 2-3 times larger values of $\rho_{\text {lin }}$ and more high values of $k_{p r s}$ and $k_{p r l}$ than ZOC. ZOC-based varistors with lower $k_{p r s}$ and $k_{p r l}$ values provide more effective surge protection than TOC-based varistors.

Keywords: varistor, $\mathrm{ZnO}$-based ceramics, $\mathrm{SnO}_{2}$-based ceramics, surge protection.

Received 03.11.2019; Received in revised form 10.12.2019; Accepted 23.12.2019

\section{Introduction}

Ceramic materials based on $\mathrm{ZnO}$ and $\mathrm{SnO}_{2}$ with the additives of other oxides have a highly non-linear current-voltage characteristic (CVC). This allows using them in the production of varistors intended for the protection of electronic and electrical equipment from overvoltage [1]. In particular, zinc oxide varistors are widely used in surge suppressors (SS) intended for protecting the power lines and electrical equipments from switching and lightning surges [2]. SS is connected in parallel to the protected object. Due to the high nonlinearity of the CVC of varistors, SS sharply reduces the resistance under the action of an overvoltage pulse. As a result, most of the overvoltage remains in the external electrical circuit and on the SS and the protected object, the voltage increases weakly.

An important parameter of varistors based on zinc oxide ceramics (ZOC) and tin oxide ceramics (TOC) is the protection coefficient $k_{p r}$. This parameter defines the level of surge protection. There are protection coefficients against the switching surge $k_{p r s}$ and the lightning surge $k_{p r l}$, which are defined as

$$
k_{\text {prs }}=\frac{E_{100}}{E_{0.001}} ; \quad k_{p r l}=\frac{E_{1000}}{E_{0.001}},
$$

where $E_{100}, E_{1000}$, and $E_{0.001}$ are electric field strengths in ceramics at the current densities 100 $\mathrm{A} \cdot \mathrm{cm}^{-2}, 1000 \mathrm{~A} \cdot \mathrm{cm}^{-2}$, and $0.001 \mathrm{~A} \cdot \mathrm{cm}^{-2}$, respectively. The current density of $0.001 \mathrm{~A} \cdot \mathrm{cm}^{-2}$ corresponds to the operating condition of the varistor in the absence of overvoltage. In this case, a classification voltage $U_{C}$ is applied to the varistor, which creates an electric field strength $E_{0.00 I}$ in the varistor material. In accordance with equation (1), the efficiency of surge protection is better as much as closer the values of $k_{p r s}$ and $k_{p r l}$ to unit.

As is known [3], the high nonlinearity of the CVC of varistor ceramics is associated with Schottky double energy barriers. Such barriers are formed in the surface region of the contacting grains of $\mathrm{ZnO}\left(\mathrm{SnO}_{2}\right)$ during the ceramics synthesis. The cause for high nonlinearity of the CVC is a reversible electrical breakdown in the near-surface region of grains where the barriers are located. This breakdown is due to the mechanisms of Zener tunneling [4] and impact ionization [5]. The consequence of such mechanisms is a high nonlinearity of $\mathrm{CVC}$ with the nonlinear coefficient $\alpha \sim 50$ in the region of classification 
voltage $U_{C}$ of varistor. It should be noted that at currents more than $1 \mathrm{~A} \cdot \mathrm{cm}^{-2}$, the nonlinear coefficient $\alpha$ decreases with increasing current density. The cause of such behavior is the comparison in value of the linear resistance in grain volume and the nonlinear resistance in surface regions of the grains where double Schottky barriers are localized. In this case, the linear resistance of grains limits the nonlinear current through Schottky barriers. Therefore, the decrease of the nonlinearity coefficient $\alpha$ takes place. Decrease of $\alpha$ leads to increasing of electric field strengths $E_{100}$ and $E_{1000}$. As a result, the protection coefficients $k_{p r s}, k_{p r l}$ increase. This reduces the efficiency of surge protection.

The purpose of this work is to study the effect of bulk electrical conductivity of grains on the protective properties of varistors based on ceramics with a highly nonlinear current-voltage characteristic.

\section{Samples and methods of investigation}

Investigations were performed on the samples of $\mathrm{ZOC}$ varistors $\mathrm{CH} 2-1$ (manufacturer "Progress" plant) and SIOV-S20K250 (manufacturer Siemens Semiconductors). Further we will denote the samples of such ceramics as Z0 and Z1, respectively. Usually, the ZOC composition for zinc oxide varistors contains 95 - 97 mol.\% $\mathrm{ZnO}$ and small additives of oxides $\mathrm{Bi}_{2} \mathrm{O}_{3}, \mathrm{Sb}_{2} \mathrm{O}_{3}, \mathrm{Co}_{3} \mathrm{O}_{4}, \mathrm{MnO}_{2}$, and $\mathrm{Cr}_{2} \mathrm{O}_{3}$.

Studies of varistor ceramics based on $\mathrm{SnO}_{2}$ were performed on the samples obtained in laboratory conditions. The composition and designation of samples for such ceramics are presented in the Table 1 .

Composition of the samples of varistor ceramics based on $\mathrm{SnO}_{2}$

Table 1

\begin{tabular}{|c|c|c|c|c|c|c|c|}
\hline \multirow{2}{*}{ Designation } & \multicolumn{7}{|c|}{ Composition, mol.\% } \\
\cline { 2 - 8 } & $\mathrm{SnO}_{2}$ & $\mathrm{CoO}$ & $\mathrm{Nb}_{2} \mathrm{O}_{5}$ & $\mathrm{Cr}_{2} \mathrm{O}_{3}$ & $\mathrm{Y}_{2} \mathrm{O}_{3}$ & $\mathrm{SrCO}_{3}$ & $\mathrm{MgO}$ \\
\hline $\mathrm{S} 1$ & 97.4 & 2.5 & 0.05 & 0.05 & 0.05 & - & - \\
$\mathrm{S} 4$ & 97.4 & 2.5 & 0.05 & 0.05 & 0.05 & 0.2 & 0.1 \\
\hline
\end{tabular}

The samples for investigation had the shape of cylinder with a diameter of base from $12 \mathrm{~mm}$ to $20 \mathrm{~mm}$ for different samples and a height (thickness) from $0.7 \mathrm{~mm}$ to $2 \mathrm{~mm}$. The bases of the cylindrical samples were used as the surface for electrodes. Electrodes were obtained by means of silver paste firing. All measurements were performed at an ambient temperature of $292 \mathrm{~K}$.

The current-voltage characteristics on the direct current were measured in the range of $10^{-6}-10^{-2} \mathrm{~A} \mathrm{~cm}^{-2}$. The samples with electrodes whose diameter was smaller by $1-1.5$ $\mathrm{mm}$ than the diameter of surfaces for electrodes were used. Currents and voltages were measured with a relative error of $\pm 2 \%$ by V7-27 digital multipurpose tester. These data were used for constructing the current-voltage characteristics of ceramic samples in the coordinates $E \sim J$ and the coordinates $\log E \sim \log J$. Here $E=U / L$ is the average value of electric field strength created by the voltage $U$ in a sample of thickness $L, J=I / S$ is the average current density in a sample with electrode area $S$ at the current $I$ through the sample. CVC were used for the determination of the electric field strength $E_{0.001}$ and the nonlinearity coefficient $\alpha_{0.001}$. The value $\alpha_{0.001}=\Delta \log J / \Delta \log E$ was found in the region of current density $J=0.001 \mathrm{~A} \cdot \mathrm{cm}^{-2}$.

Investigations of the CVC at $J>10 \mathrm{~A} \cdot \mathrm{cm}^{-2}$ were performed on single voltage pulses with exponential form. In this case, we used the samples with small electrodes (area of $0.025-0.04 \mathrm{~cm}^{2}$ ) to obtain large values of current density. Techniques for creating small electrodes and studying the CVC at high currents are described in [6]. Oscillograms of 
voltage and current during pulse tests were recorded using a double-beam storage oscilloscope C8-11.

For registering the current-voltage characteristics at high electric currents with a relative error not more than $\pm 1.5 \%$, raster images of oscillograms were used. Such images were obtained by means of photographing oscillograms from the oscilloscope screen by the digital camera OLYMPUS C-756. The values of current and voltage at various points of the CVC were determined on the basis of data obtained at scanning the coordinates of raster image by means of the program described in [7]. The CVC obtained in this way were used to determine the electric field strengths $E_{100}, E_{1000}$ and nonlinearity coefficients $\alpha_{100}, \alpha_{1000}$ at the current density of $100 \mathrm{~A} \cdot \mathrm{cm}^{-2}$ and $1000 \mathrm{~A} \cdot \mathrm{cm}^{-2}$, respectively.

The effective specific linear resistance $\rho_{l i n}$, which limits the current of intergranular barriers of varistor ceramics in the region of current density $J$, was determined with an error of $\pm 4 \%$ from raster images of oscillograms of current and voltage. The technique for measuring the $\rho_{\text {lin }}$ was based on the differential resistance conception described in $[8,9]$. When performing measurements of $\rho_{\text {lin }}$, we used the program presented in [10] for processing raster images of oscillograms. The dependences $\rho_{\text {lin }}(J)$ obtained in the region of high currents were used to determine the effective linear resistances $\rho_{\operatorname{lin} 100}$ and $\rho_{\operatorname{lin} 1000}$ at the current density of $100 \mathrm{~A} \cdot \mathrm{cm}^{-2}$ and $1000 \mathrm{~A} \cdot \mathrm{cm}^{-2}$, respectively.

\section{Experimental results and discussion}

The parameters for different samples of varistor ceramics studied in this work are presented in Tab. 2. As can be seen, near the electric current density of $0.001 \mathrm{~A} \cdot \mathrm{cm}^{-2}$, all samples have a nonlinear coefficient of the current-voltage characteristic $\alpha_{0.001}$ not less than 30. In the region of high current, value of $\alpha$ decreases with current increasing. The cause for this is the limitation of current through intergranular barriers by the linear bulk resistance of grains in varistor ceramics. Current limitation occurs when all barriers in the current path between the sample electrodes operate in the regime of reversible electric breakdown caused by Zener tunneling and avalanche breakdown [4, 5].

As shown in $[11,12]$, the disordering of varistor ceramics associated with a spread in grain sizes and heights of intergranular barrier leads to current localization in the percolation paths. When all barriers in percolation path are in the breakdown regime, this path has a low resistance and shunts paths containing at least one barrier not functioning in the breakdown regime. Just for such percolation paths, the current of intergranular barriers is limited by the linear bulk resistance of the grains. Such paths determine the $\mathrm{CVC}$ of varistor ceramics in the region of high electric currents. Localization of current in the percolation paths indicates that electric current is unevenly distributed over the cross section of ceramic sample.

Table 2

Parameters of varistor ceramics based on zinc oxide $(\mathrm{Z0}, \mathrm{Z1})$ and tin dioxide $(\mathrm{S1}, \mathrm{S2})$

\begin{tabular}{|c|c|c|c|c|c|c|c|c|c|c|c|c|}
\hline & \multirow{2}{*}{$\begin{array}{c}E_{0.001} \\
\mathrm{~V} \cdot \mathrm{cm}^{-1}\end{array}$} & \multirow{2}{*}{$\begin{array}{c}E_{100,}, \\
\mathrm{~V} \cdot \mathrm{cm}^{-1} \\
\end{array}$} & \multirow{2}{*}{$\begin{array}{c}E_{1000} \\
\mathrm{~V} \cdot \mathrm{cm}^{-1}\end{array}$} & \multirow{2}{*}{$\alpha_{0.001}$} & \multirow{2}{*}{$\alpha_{100}$} & \multirow{2}{*}{$\alpha_{1000}$} & \multirow{2}{*}{$\begin{array}{l}\rho_{\text {lin } 100,} \\
\Omega \cdot \mathrm{cm}\end{array}$} & \multirow{2}{*}{$\begin{array}{c}\rho_{\text {lin } 1000,} \\
\Omega \cdot \mathrm{cm}\end{array}$} & \multicolumn{2}{|c|}{ experiment } & \multicolumn{2}{|c|}{ calculation } \\
\hline & & & & & & & & & $k_{p r c}$ & $k_{p r s}$ & $k_{p r c}$ & $k_{p r s}$ \\
\hline $\mathrm{Z} 0$ & 1512 & 2148 & 2582 & 42 & 10 & 4.3 & 2.35 & 0.6 & 1.42 & 1.71 & 1.47 & 1.79 \\
\hline $\mathrm{Z} 1$ & 1672 & 2355 & 2941 & 52 & 10.5 & 4.8 & 2.05 & 0.58 & 1.41 & 1.76 & 1.37 & 1.65 \\
\hline S1 & 2280 & 3770 & 4650 & 30 & 6.5 & 3.8 & 5.72 & 1.29 & 1.64 & 2.04 & 1,72 & 2,15 \\
\hline S4 & 2730 & 3985 & 5241 & 54 & 6.3 & 3.6 & 6.80 & 1.67 & 1.46 & 1.92 & 1.49 & 1.90 \\
\hline
\end{tabular}

The distribution of the grain sizes in ceramics is the reason that different percolation paths between electrodes of a sample contain different number of grain boundaries with barriers. At increase of the voltage, at first the reversible breakdown takes place for 
percolation paths with smallest number of barriers. With the increase of voltage the number of percolation paths grows.

The effective linear resistivity $\rho_{\text {lin }}$ measured in the region of current density $J$ is determined by the number of percolation paths formed at this current density. From the data of Tab. 2 it follows, that the decrease of $\rho_{\text {lin }}$ takes place with increasing of $J$. This is a consequence of the fact that with increasing voltage (current density) the increase of the number of parallel percolation paths. At the currents of about $1000 \mathrm{~A} \cdot \mathrm{cm}^{-2}$ or more, almost all possible current paths between the electrodes of a sample contain only intergranular barriers operating in the nonlinear regime. Therefore, $\rho_{\text {lin }}$ is close to the specific bulk resistance of the grains in varistor ceramics $\rho_{g}$. In Tab. 2 the value of $\rho_{\text {lin } 1000}$ is close to the value of $\rho_{g}$ for different compositions of varistor ceramics.

The relationship between the parameters of varistor ceramics (Tab. 2) and the protection coefficients can be obtained. For the description of the current-voltage characteristic of intergranular barriers, we use the empirical dependence between the electric field strength $E$ and the electric current density $J[1]$ :

$$
J=B E^{\alpha}
$$

where $B$ is a constant and $\alpha$ is the nonlinearity coefficient of CVC.

If we take into account the values of nonlinearity coefficient of CVC $\alpha_{0.001}$ and the electric field strength $E_{0.001}$ and also assume that $\alpha=\alpha_{0.001}$, then the expression (1) can be written in the form

$$
J=0.001\left(\frac{E}{E_{0.001}}\right)^{\alpha_{0.001}} .
$$

It follows from (3), the average electric field strength $E_{b}$ associated with a voltage drop on the grain boundaries in ceramics is determined as

$$
E_{b}=E_{0.001}\left(\frac{J}{0.001}\right)^{\frac{1}{\alpha_{0.001}}} .
$$

At the weak currents almost all voltage $U$ applied to a sample drops in the grain surface regions with high resistance where barriers are localized. Therefore, the average electric field strength in ceramics is $E=E_{b}$. At high currents the part of the voltage $U$ applied to the bulk resistance of grains becomes comparable to the voltage drop in the surface region of grains. This contribution $E_{g}$ to the electric field strength $E$ can be represented as $E_{g}=J \rho_{\text {lin }}$. Since $E=E_{b}+E_{g}$ for the CVC of varistor ceramics in the region of high electric currents, the following empirical expression can be obtained:

$$
E=J \rho_{l i n}+E_{0.001}\left(\frac{J}{0.001}\right)^{\frac{1}{\alpha_{0.001}}}
$$

From (1) and (5) for protection coefficients $k_{p r s}$ and $k_{p r l}$ one can obtain:

$$
k_{\text {prs }}=\frac{100 \rho_{\text {lin } 100}}{E_{0.001}}+10^{\frac{5}{\alpha_{0.001}}},
$$




$$
k_{p r l}=\frac{1000 \rho_{\operatorname{lin} 1000}}{E_{0.001}}+10^{\frac{6}{\alpha_{0.001}}},
$$

In the Table 2 the values of coefficients $k_{p r s}$ and $k_{p r l}$ calculated by (1) using the experimental $E_{100}$ and $E_{1000}$ and the values of these coefficients calculated by (6), (7) using $\rho_{\text {lin } 100,}, \rho_{\text {lin1000 }}, E_{0.001}, \alpha_{0.001}$ are shown. As can be seen, there is a correspondence between the experimental and calculated values of $k_{\text {prs }}$ and $k_{p r l}$.

As follows from the relations (6) and (7), values of $k_{p r s}$ and $k_{p r l}$ increase with increasing values of $\rho_{\text {lin } 100}$ and $\rho_{\text {lin } 1000}$. This means that at the same values of parameters $\alpha_{0.001}$ and $E_{0.001}$ the efficiency of surge protection for varistors based on ceramics with large values of $\rho_{\text {lin }}$ is worse than for varistors with lower values of $\rho_{\text {lin }}$. Since the value of $\rho_{l i n}$ is determined by the bulk resistivity $\rho_{g}$ of grains, the efficiency of surge protection can be improved by the value decrease of $\rho_{g}$. This can be realized by finding oxide additives to ceramic composition that reduce $\rho_{g}$ without decreasing the nonlinearity of CVC.

According to (6), (7), the protection coefficients $k_{p r s}, k_{p r l}$ have minimum values when $\rho_{\text {lin }}=0$. These values are determined by expressions:

$$
k_{p r s(\min )}=10^{\frac{5}{\alpha_{0,001}}} ; \quad k_{p r l(\min )}=10^{\frac{6}{\alpha_{0,001}}} .
$$

At $\alpha_{0,001}=50 k_{p r s}=1.26, k_{p r l}=1.32$. It is possible to reduce $k_{p r s}, k_{p r l}$ and improve the surge protection by increasing the nonlinearity coefficient $\alpha_{0.001}$.

The effective linear resistivity $\rho_{\text {lin }}$, which limits the current of barriers in varistor ceramics, increase the minimum values of the protection coefficients $k_{p r s(\min )}, k_{p r l(m i n)}$, on the quantity $100 \rho_{\text {lin }} / E_{0.001}$ and $1000 \rho_{\text {lin }} / E_{0.001}$, respectively. The magnitude of this additive decreases with an increase of $E_{0.001}$. Therefore, for varistors based on the ceramics with relatively small values of $E_{0.001}$, one should expect larger values of $k_{p r s}$ and $k_{p r r}$. Such varistors provide less effective protection against overvoltage than varistors with larger values of $E_{0.001}$.

Based on the data presented in Tab. 2, we can conclude that varistor ceramics based on $\mathrm{SnO}_{2}$, though it has a high nonlinearity coefficient $\alpha_{0.001}$, its values of $\rho_{\text {lin }}$ are approximately 2-3 times larger. The results of this are the higher values of protection coefficients $k_{p r s}, k_{p r l}$. Therefore, at present, TOC based varistors are less effective for surge protection than ZOC based varistors. To improve the protective properties of TOC varistors, it is necessary to search the ceramic compositions with lower effective linear resistivities $\rho_{l i n}$, which limits the current of intergranular barriers.

\section{Conclusions}

The current-voltage characteristics of the nonlinear varistor ceramics based on $\mathrm{ZnO}$ (ZOC) and $\mathrm{SnO}_{2}$ (TOC) of different compositions were studied in a wide range of electric current. On the basis of obtained data the coefficients $k_{p r s}=E_{100} / E_{0.001}$ and $k_{p r}=E_{1000} / E_{0.001}$ were determined; they characterize the level of protection against switching surge and lightning surge ( $E_{0.001}, E_{100}$, and $E_{1000}$ are electric field strengths at the current density of $0.001 \mathrm{~A} \cdot \mathrm{cm}^{-2}, 100 \mathrm{~A} \cdot \mathrm{cm}^{-2}$ and $1000 \mathrm{~A} \cdot \mathrm{cm}^{-2}$, respectively). The lower the values of these coefficients are, the more effective the surge protection is. A relationship between $k_{p r s}, k_{p r l}$ and the effective linear resistivity $\rho_{\text {lin }}$ limiting the current of intergranular barriers in varistor ceramics was established. It was shown that the values of $k_{p r s}$ and $k_{p r l}$ increase 
with increasing value of $\rho_{\text {lin }}$, the magnitude of which is determined by the bulk resistance of grains in ceramics. The tin oxide ceramics have values of $\rho_{\text {lin }} 2-3$ times greater than for the zinc oxide ceramics and, as a result, larger values of $k_{p r s}$ and $k_{p r l}$. Therefore, ZOC based varistors with lower coefficients of $k_{p r s}$ and $k_{p r l}$ provide more effective surge protection than TOC based varistors.

\section{References}

1. Gupta, T.K. Application of zinc oxide varistors // Journal of the American Ceramic Society. - 1990. - Vol. 73, No. 7. - P. $1817-1840$.

2. Dmitriev, M.V. Use of arresters to protect the insulation of overhead lines 6-750 kV. - SPb.: Polytechnic university publishing house, 2009. - 92 p. (in Russian).

3. Clarke, D.R. Varistor ceramics // Journal of the American Ceramic Society. 1999. - Vol. 82, No. 3. - P. 485 - 502.

4. Eda, R. Conduction mechanism of non - Ohmic zinc oxide ceramics // Journal of Applied Physics. - 1978. - Vol. 49, No. 5. - P. 2964 - 2972.

5. Pike, G.E. Electroluminescence in $\mathrm{ZnO}$ varistors: Evidence for hole contributions to the breakdown mechanism / G.E. Pike, P.L. Gourley // Journal of Applied Physics. 1985. - Vol. 57, No. 4. - P. 5512 - 5518.

6. Lu, Z.Ya. Electrical properties of new tin dioxide varistor ceramics at high current / Z.Ya. Lu, A.B. Glot, A.I. Ivon, Z.Ya. Zhou // Journal of the European Ceramic Society. - 2012. - Vol. 32. - P. $3801-3807$.

7. Ivon, A.I. Software for processing digital images of analog oscillograms / A.I. Ivon, V.F. Istushkin // System Technologies. - 2018. - Issue 1' (114). - P. 45 - 52.

8. Ivon, A.I. Parameters of $\mathrm{ZnO}$ grains in $\mathrm{ZnO}$-based ceramics // Inorganic Materials. - 2000. - Vol. 36, No. 10. - P. $1074-1077$.

9. Ivon, A.I. High-current measurement of the grain resistivity in zinc oxide varistor ceramics / A.I. Ivon, R.I. Lavrov, A.B. Glot // Ceramics International. - 2013. - Vol. 39(6). - P. $6441-6447$.

10. Ivon, A.I. Software for determining linear resistance limiting the barrier current in varistor materials / A.I. Ivon, Y.A. Ivon, R.I. Lavrov // System Technologies. - 2017. Issue 1 (108). - P. $29-36$.

11. Vojta, A. Influence of microstructural disorder on the current transport behavior of varistor ceramics / A. Vojta, Q. Wen, D. R. Clarke // Computational Materials Science. - 1996. - Vol. 6, No. 1. - P. $51-62$.

12. Bartkowiak, M. Voronoi network model of $\mathrm{ZnO}$ varistors with different types of grain boundaries / M. Bartkowiak, G. D. Mahan, F.A. Modine, M.A. Alim, R. Lauf, A. McMillan // Journal of Applied Physics. - 1996. - Vol. 80, No. 11. - P. 6516 - 6522 